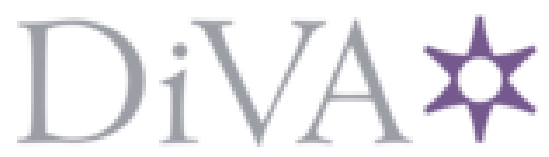

http://www.diva-portal.org

This is the published version of a paper presented at Image and Signal Processing (CISP), 2012.

Citation for the original published paper:

Wei, W., Khatibi, S. (2012)

A New Methodology for Evaluating Various Methods of 3D Reconstruction from Single Image.

In: (pp. 582-586). IEEE

http://dx.doi.org/10.1109/CISP.2012.6469872

N.B. When citing this work, cite the original published paper.

Permanent link to this version:

http://urn.kb.se/resolve?urn=urn:nbn:se:bth-11170 


\section{A New Methodology for Evaluating Various Methods of 3D Reconstruction from Single Image}

\author{
Wei Wen \\ School of Computing \\ Blekinge Institute of Technology \\ Karlskrona, Sweden
}

\author{
Siamak Khatibi \\ School of Computing \\ Blekinge Institute of Technology \\ Karlskrona, Sweden
}

\begin{abstract}
Consumer market is recently experiencing 3D display. To prepare the market for new era of 3D TV, the 3D display popularity can be increased by making ease of capturing or generating of stereo information. The results of recent research on 3D structure modeling from single images can be used to generate stereo images. We evaluate some of typical 3D reconstruction methods from single images in relation of using the results in 3D display. The evaluation is not only based on the experts' opinions and statistic test but also an objective methodology is introduced. Only planar surfaces, like as in any man made structure scenes, are used in the objective evaluation. The number of planes is varied in a controlled way. Three popular and classic methods are chosen for the evaluation with this new methodology. And the evaluation result is consistent with the statistical result of human inquiries.
\end{abstract}

Keywords-component; 3DTV; evaluation; $3 D$ reconstruction; single image

\section{INTRODUCTION}

Nowadays the 3D TV does not seem far away considering mass production of $3 \mathrm{D}$ display and its introduction to consuming market. The 3D display is still using glasses despite of that glass-free 3D display technology exists and advancing to its final matureness. Both of these technologies are depending on depth information using stereo or multiple images as their input materials. In this sense the possibility of using a single image as a source of stereo information is very attractive especially when there are not two or more images prepared for 3D reconstruction. Despite of that the process is an ill-posed problem; the decade of research has shown it is possible to extract the depth information or reconstruct a 3D model. For this purpose a variety of methods has been developed which can be classified with respect to their major directions such as depth reconstruction-, image content-, and psychology- based methods. In other words, these methods are based on different cues.

However simple observation by human eyes and personal feelings cannot show the effectiveness of the methods for solving the problem. For example, some methods are more effective for urban images, but work badly on the natural scenes. As far as more and more persons are aware of that there is no objective comparison for such methods, in other words there is no standard of comparison methodology for this purpose. In this paper, we try to show it can be possible to obtain a methodology for evaluating such vast types of methods in solving the same problem which is $3 \mathrm{D}$ reconstruction from single image. In the previous works, the evaluation is usually only based on few human inquires. People just focus on their favorite parts of the 3D result based on their interest and experience. Saxena et al. defined the final result as correct when $70 \%$ of the major planes in the image (the major plane occupies more than $15 \%$ of the image) are in correct relationship with their nearest neighbors [1]. Olga Barinova et al. gathered about the $3 \mathrm{D}$ results, and then they arrived at a conclusion that which method is the best [2]. But it is obvious that the personal inquiries are not sufficient and objective for evaluating the $3 \mathrm{D}$ results from different $3 \mathrm{D}$ reconstruction methods. Evaluating methods based on human ratings has its advantage that we obtain a ground truth. However, they are not always applicable, especially in automated systems.

In this paper, a new and objective method is introduced to evaluate and compare in term of the various $3 \mathrm{D}$ reconstruction methods from single images. Several pictures from man-made scenes were captured where the scene were built on purpose. The pictures were used and 3D models were obtained implementing the chosen methods. In these simple scenes, the details are given, such as the number of planes and the distance between each plane and the camera. Then it is easy to find out the same and different parts between the $3 \mathrm{D}$ result and the real $3 \mathrm{D}$ scene, which is helpful to check whether the method is effective or not. Later on, human inquiries were also done and analyzed statistically for correlating the two methodologies are to find out how consistent they are to each other.

\section{BACKGROUND AND METHODS}

From the eighties of the last century more and more methods showed up for $3 \mathrm{D}$ reconstruction from a single still image. More and more clues have been found which are used for $3 \mathrm{D}$ reconstruction. Criminisi Reid and Zisserman first defined the vanishing line and vanishing point. The vanishing line is determined by the two vanishing points which are the intersections of the lines in the image that are parallel lines in reality [3]. According to this they calculated the angles between parallel lines, relationships between planes and then inferred the 3D structure. The cues such as shading and texture are used for the methods such as shape from shading [4] and 
shape from texture [5] normally which can get good results from uniform color or texture, but show poor results when processing the image with complex color or texture. Peter Kovesi reconstructed the shape of the object from its surface normal so called shapelets [6], which is very simple to implement and robust to noise. Using mathematic model is introduced in the 3D reconstruction methods. Delage, Lee, and $\mathrm{Ng}$ [7] built the 3D model of indoor scenes which only contains the vertical walls and ground from single image based on the model using the dynamic Bayesian network. The model needed the prior knowledge of the environment. Felzenszwalb and Huttenlocher [8] developed a method for the image segmentation based on the content of the image. It was the first time where the superpixel was defined which nowadays becomes a foundation for the many methods of $3 \mathrm{D}$ reconstruction.

But the performance evaluation for above methods are based on human inquiry and rating. Human perception is one of the most important measurements for images. However, if one method need to be applied for industry and real life, the objective evaluation would still be necessary.

According to the classification with respect to their major directions, the following three methods are chosen to be evaluated by the proposed methodology:

a) 3D Depth Reconstruction from a Single Still Image. ("Make3D" for short) [9].

b) Automatic photo pop-up. ("Popup" for short) [10].

c) Stereo image displaying based on both physiological and psychological stereoscopy from single image. ("Psych" for short) [11].

The reason why to choose these methods are that these three methods represented several types of methods for 3D reconstruction. Make3D represented the method which depth in different ways is estimated; the method of Popup is partly based on image content; the Psychological method is obviously based on the human's psychology and both eyes and brain functioning.

\section{A. Make3D}

In this method, both the 3D location and the orientation of the small planar regions in the image are inferred using a Markov Random Field (MRF). In the method "the relation between the image features and the location/orientation of the planes is based on a learning. Also the relationships between various parts of the image are found using supervised learning (supervised learning is a machine learning technique to predict the value of a function which is obtained from training data. The training data consists of pairs of input objects and desired outputs.)". The basic clue here is called 'superpixel' which in its turn is related to the location/orientation of the planes. The desired outputs in the training data are obtained by the 3D laser scanner. A data base which consists of various types of scenes is created first, and then the depth maps of these scenes can be detected by this equipment. Depending on the likelihood of superpixels map and scanner's depth map, it is easy to build the $3 \mathrm{~d}$ structure from a $2 \mathrm{D}$ image. The step on the calculation of superpixels' likelihood is called MAP inference.

\section{B. Automatic photo pop-up}

In this method a virtual $3 \mathrm{D}$ structure from a single still image is created completely automatically. It looks like as the image is laid on the ground and then the areas that should be perpendicular to the ground are 'popped up' as vertical planes. One of the goals is to achieve a fast $3 \mathrm{D}$ reconstruction.

The method assumes that input images are from outdoors scenes including both natural and man-made (buildings) and also assumes that each scene consists of a single ground plane, and piece-wise planar objects sticking out of the ground at right angles, and there is also sky in the scene. Under these assumptions, it builds a coarse, scaled 3D model from a single image by classifying each pixel into three labels which are ground, vertical or sky and estimating the horizon position. Color, texture, objects location, and geometric features are all useful cues for determining these labels.

\section{Psychological and Physiological stereoscopy}

Usually, human perceives the $3 \mathrm{~d}$ subjects with two eyes; it is easy to get the information of everything. But with only one eye, it will be hard and even impossible to perceive the depth and other $3 \mathrm{D}$ information in natural way of an image. So in this method they try to create a new image from the original one. With the two images, people can easily build the $3 \mathrm{~d}$ structure based on the human's psychology and physiological function assuming to observe each image by only one eye.

The depth perception by brain is based on stereoscopy. There are two kinds of human's stereoscopy. One is psychological stereoscopy, which is based on the visual memory and experience. It means that, when people see an image they could understand the 3D content (depth) with help of clues which are stimulated by the image and it is not hard for them to orientate different objects in depth. These cues or hints are some kind of experience and memory in human's brain, which are collected by people in a long period of time. So human beings can perceive the depth and position of objects in one image with their own memory and experience. The other one is physiological stereoscopy. This is based on structure and physical functionality of the eye. Binocular disparity is the most important element of physiological stereoscopy. It has been proved that after excluding all the psychological elements, it is possible that a set of visual stimulations can create the depth feeling with two eyes under the binocular disparity condition [11]. Due to the depth disparity of binocular stereoscopy the $3 \mathrm{D}$ content is perceived which also important in stereo imaging. Usually both psychological stereoscopy and physiological stereoscopy give a person the same clue of an image and enhance the human's stereoscopy seeing, which is the basic idea of this method.

\section{METHODOLOGY}

By observing and comparing the procedures of the chosen methods, common steps in the method could be found which 
we could implement them to find a novel objective methodology for evaluating and comparison of the results e.g. the methods attempt to find the right ground plane and the right number of vertical planes in the single image. Especially in urban scenes, the primary objective of the methods is to fix the buildings and walls' positions which are all perpendicular to the ground. Even in the natural scenes, the hills, rocks and trees can also be considered as vertical which also means that the proposed methodology can be beneficial. Thus finding the right ground plane and vertical planes are more important and common goals in the methods which should be detected and modeled. Based on this conclusion, a set of simple scenes which each scene is composed by only a ground plane and several vertical planes were created and the scene was captured as an image.

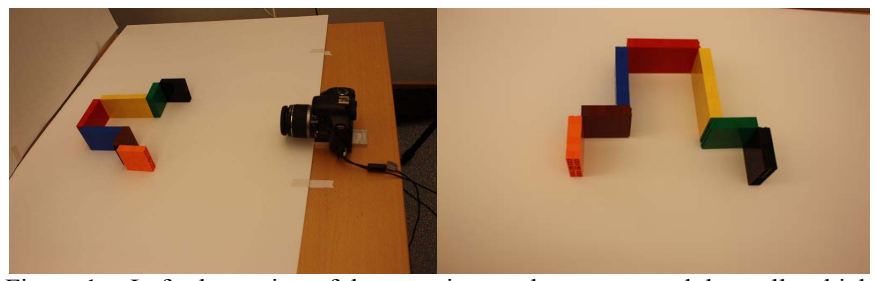

Figure 1. Left: the setting of the experiment, the camera and the walls which were made from Lego toy. Right: one of the original images

In Figure 1, several walls were made from Lego toys with the same height and different width, and there are only one ground plane and seven walls in the scene. Each wall is perpendicular to the ground plane and conceited to the neighboring wall; the camera is also parallel to the ground and perpendicular to the walls. In each scene we had control on the complexity of the scene by using more walls and in different depth. To compare the methods different scenes were created with different complexities. As far as each scene was designed in a controlled way it is possible to compare the $3 \mathrm{D}$ result of the methods with each other for each captured image from a scene.

\section{RESULTS}

\section{A. Result of the novel objective methodology}

The images of these scenes which contains two, three, five or six vertical planes were captured and then they were processed by the above three methods. For the psychological and physiological method the disparity map as the result was computed to find out the detected number of planes. In figure2, three groups of results are shown and in table 1, we show the numbers of planes in the $3 \mathrm{D}$ results of each method when there are different numbers of planes in the real scenes.

\section{B. Results of personal evaluation inquiries}

1) Rules for personal evaluation inquiries

The results of the $3 \mathrm{D}$ reconstruction would be evaluated by personal inquires which is depending on the people's feelings and experience. The inquiries would be analyzed statistically. During inquiry 20 persons with different backgrounds (from different countries, different educational levels and different ages) participated and they were able to score the results between 1 to 5 points. Each scoring point was defined as following: In the $3 \mathrm{D}$ result

a) The right angle and relative position between each two objects are achieved,

b) The right ground plane, the right horizon are found,

c) Clarity of depth perception for different objects in a scene is achieved,

d) The shape of each object is fine, and is not distorted,

e) It is possible to find as many details as there are in the scene.

The top score is 5 and score 1 represents poor $3 \mathrm{D}$ effect. For simplification and understanding of the evaluation procedure some of the models were shown to the test persons (before their evaluation) and the score definitions were explained.

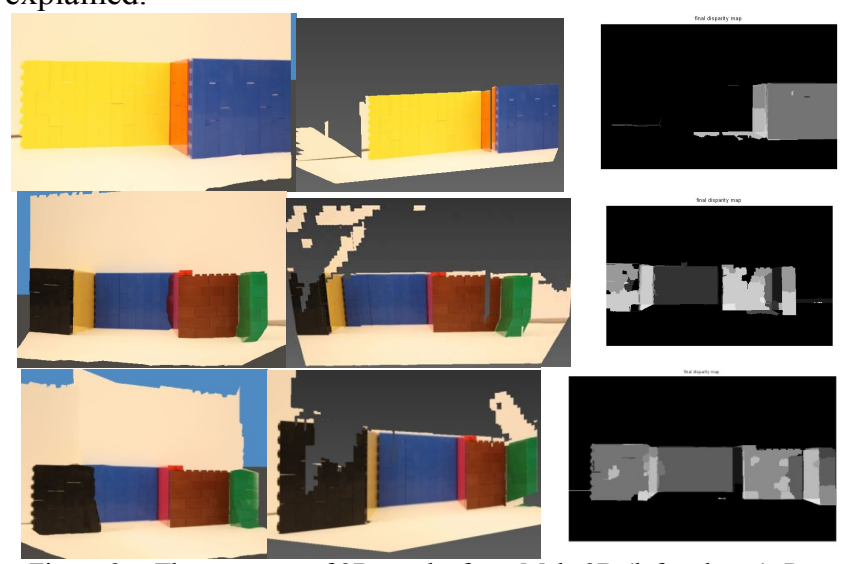

Figure 2. Three groups of 3D results from Make3D (left column), Popup (middle column), and Disparity map of Psych (right column).

TABLE I. THE COMPARISON OF THE 3D RESULTS OF THE CHOSEN METHODS BY THE NOVEL OBJECTIVE MEHTOD: THE NUMBER OF REAL PLANES AND FROM IN 3D RESULTS FOR EACH METHOD IS SHOWN.

\begin{tabular}{|c|c|c|}
\hline $\begin{array}{l}\text { Reconstruction } \\
\text { Methods }\end{array}$ & $\begin{array}{c}\text { The number of real } \\
\text { planes }\end{array}$ & $\begin{array}{c}\text { The number of planes in } \\
3 D \text { results }\end{array}$ \\
\hline Make3D & 6 & 5 \\
\hline Popup & 6 & 3 \\
\hline \multirow[t]{2}{*}{ Psych } & 6 & 6 \\
\hline & $\begin{array}{c}\text { The number of real } \\
\text { planes }\end{array}$ & $\begin{array}{c}\text { The number of planes in } \\
3 D \text { results }\end{array}$ \\
\hline Make3D & 5 & 4 \\
\hline Popup & 5 & 3 \\
\hline \multirow[t]{2}{*}{ Psych } & 5 & 5 \\
\hline & $\begin{array}{c}\text { The number of real } \\
\text { planes }\end{array}$ & $\begin{array}{c}\text { The number of planes in } \\
3 D \text { results }\end{array}$ \\
\hline Make3D & 3 & 3 \\
\hline Popup & 3 & 2 \\
\hline \multirow[t]{2}{*}{ Psych } & 3 & 3 \\
\hline & $\begin{array}{c}\text { The number of real } \\
\text { planes }\end{array}$ & $\begin{array}{c}\text { The number of planes in } \\
3 D \text { results }\end{array}$ \\
\hline Make3D & 2 & 2 \\
\hline Popup & 2 & 1 \\
\hline Psych & 2 & 2 \\
\hline
\end{tabular}




\section{2) Database}

A database including 80 images was created where 30 of images are about natural scenes, another 30 ones are about urban scenes, and the rest of 20 images are about indoor scenes. The natural images are consisted of the beach, cliff, road and field scenes and so on. The indoor images include many kinds of images which were taken inside a building; even an image is so simple that scene is only one bowl. Thus the database contains different kinds of images from different kinds of environments. All of the images are either downloaded from the internet or taken by the authors of this paper and picked out randomly.

\section{3) Results}

Three groups of $3 \mathrm{D}$ reconstruction results prepared for human inquiries are shown in figure 3 and the average scores of the methods are shown in table 2 which are calculated by all the marking results from the volunteers and for different types of images.

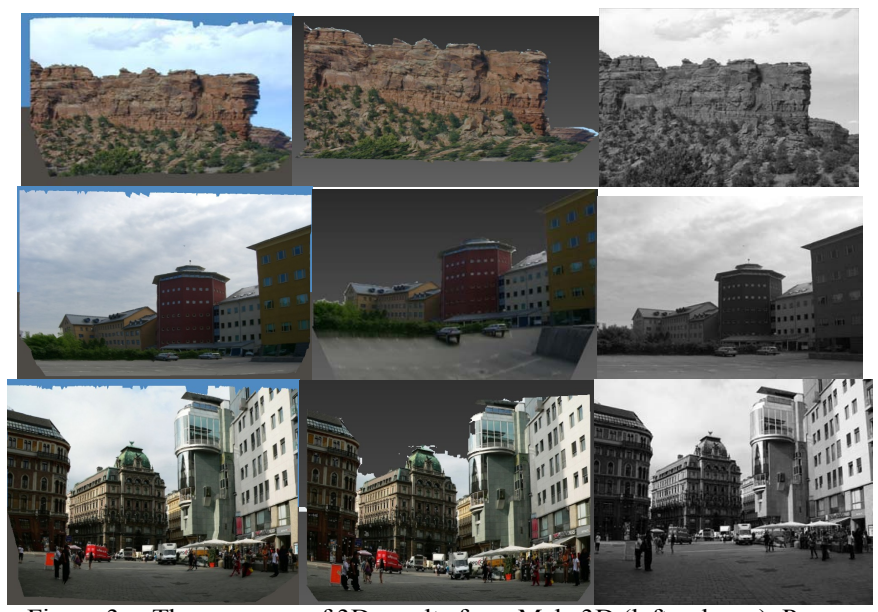

Figure 3. Three groups of 3D results from Make3D (left column), Popup (middle column), and Disparity map of Psych (right column) for personal observation.

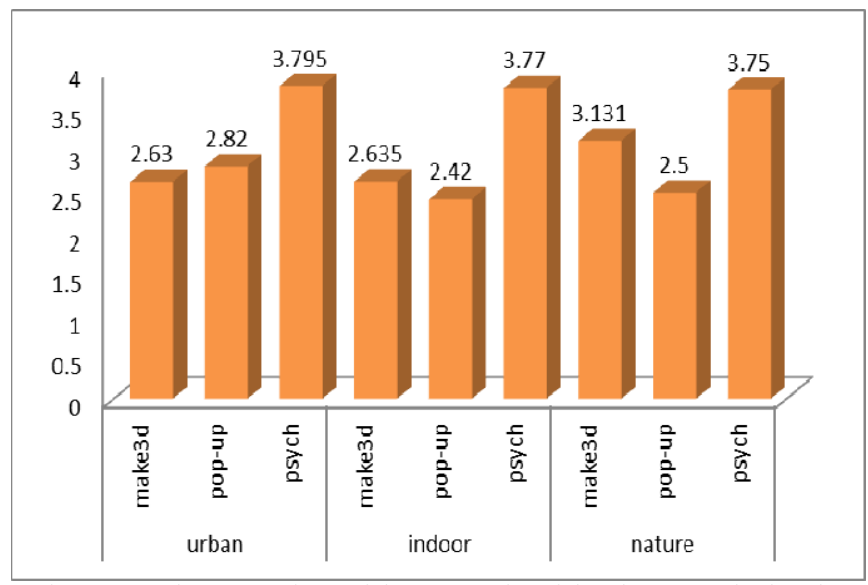

Figure 4. The comparison of the 3D results of the chosen methods: The average scores of the methods and for different type of images are shown.

TABLE II. THE PROCESSING TIME OF EACH METHOD.
\begin{tabular}{|c|c|}
\hline & $\begin{array}{c}\text { processing time } \\
\text { (second) }\end{array}$ \\
\hline Make3d & $60-120$ \\
\hline Popup & $20-40$ \\
\hline Psychology & $1-3$ \\
V. DISCUSSION
\end{tabular}

In the results shown in Table 1 , it is very clear that the psychological and physiological method create the same number of planes in the $3 \mathrm{D}$ results as the real scenes. Make3D got almost the same number of planes and the popup method lost a few planes. In the results of psychological method (the disparity map), every plane is clear and not distorted. But in the results of make3D, many parts look weird.

According to the results from human inquiries the method based on psychology and physiology is the best for all kind of images. The method, make3d receives the second place for the indoor and nature scenes. The method pop-up has the third place when it is used for all images. Because personal evaluation inquiry depends partially on human feeling, therefore the psychological and physiological method had a little extra advantage in the test. However this method is very easy to be used and computed, it does not depend on any statistical model and line-plane computation, and it does not need to determine any planes or lines. Also it can be used for any kind of images either easy scenes or complex environments. Human beings have strong feelings on the shape of buildings and rocks and also the depth of the scene. Not only this is partially depends on people's experience and psychology, but also this method is able to convert the single view image to multi-view images where the appropriate shift of pixels is needed.

In an image, there is always a significant object or an area which is the most interesting to people, this object or area motivate people to ignore the others. For example, in urban scenes the buildings are usually the most important objects and the other objects such as vehicles, people and other objects in the image are somehow not considered. In nature scenes, the hills, the rocks and trees are main part of one image. So when persons estimate a $3 \mathrm{~d}$ structure, this part will be noticed first. It means that all the $3 \mathrm{~d}$ structures which are reconstructed from a single image, still cannot cover all details in an image. What normally people do in such inquiry is to evaluate the reconstruction of everything in the scene, which was also the fifth rule in the scoring method. In the methodology presented in this paper, the simple scene was man-made and all the details were controllable. Then the colorful walls were the most significant part in the scene. What the persons need to focus on are only these walls. So it is easy for persons to compare every result from different methods.

In another Lego experiment, all the Lego walls were made to be parallel to each other and perpendicular to the camera. The distance between each plane and the camera is different, from five to fifteen centimeters. Then we obtained the 3D results with the three methods, Make3d, Popup and Psychology. In the $3 \mathrm{D}$ results from Make3d and Popup, there is 
not any depth information. All the planes are combined to one plane, which proves it is still impossible for single image to exact the depth information accurately. Some other clues are still necessary. In the $3 \mathrm{D}$ results of Psychological method, the disparity map is also not clear and not all the depths can be distinguished.

\section{CONCLUSION AND FUTURE}

A new objective evaluation methodology is presented in this paper, which is helpful for persons to compare different 3D reconstruction methods. With this methodology, we arrived at the conclusion: in these methods, psychological method is the best one, it works fast and the results are impressive; the make3d takes the second place of the methods for natural images. And this result is consistent with the result of human inquires.

This methodology is only used for evaluating the methods not for the $3 \mathrm{D}$ results. In the future we will try to evaluate the $3 \mathrm{D}$ results with a novel objective methodology not the human inquires only, which can help persons to get the better 3D results from the same method.

\section{REFERENCE}

[1] Ashutosh Saxena, Min Sun, Andrew Y. Ng (2007). Learning 3-D Scene Structure from a Single Still Image. ICCV workshop on 3D Representation for Recognition (3dRR-07).

[2] Olga Barinova, Vadim Konushin, Anton Yakubenko, KeeChang Lee, Hwasup Lim, and Anton Konushin. Fast Automatic Single-View 3-d
Reconstruction of Urban Scenes. ECCV 2008, Part II, LNCS 5303, pp. $100-113$.

[3] A. Criminisi, I. Reid, and A. Zisserman. Single view metrology. IJCV, 40:123-148, 2000

[4] R. Zhang, P.-S. Tsai, J.E. Cryer, and M. Shah. Shape from shading: A survey. IEEE Trans on Pattern Analysis and Machine Intelligence (PAMI), 21(8):690-706, 1999.

[5] J. Malik and R. Rosenholtz. Computing local surface orientation and shape from texture for curved surfaces. International Journal of Computer Vision (IJCV), 23(2):149-168, 1997.

[6] Peter Kovesi, Shapelets Correlated with Surface Normals Produce Surfaces. 10th IEEE International Conference on Computer Vision. Beijing. pp 994-1001. 2005.

[7] E. Delage, H. Lee, and A.Y. Ng. A dynamic Bayesian network model for autonomous $3 \mathrm{D}$ reconstruction from a single indoor image. In Computer Vision and Pattern Recognition (CVPR), 2006

[8] P. Felzenszwalb and D. Huttenlocher. Efficient graph-based image segmentation. Int. Journal of Computer Vision 59, 2, 167-181 2004.

[9] A.Saxena, S. H. Chung, and A. Y. Ng (2007). 3-d depth reconstruction from a single still image. In International Journal of Computer Vision(IJCV) Aug 2007.

[10] Derek Hoiem, Alexei A. Efros and Martial Hebert. Automatic Photo Pop-up. ACM SIGGRAPH 2005.

[11] Chunping Hou, Jiachen Yang, Zhuoyun Zhang. Stereo Image Displaying Based on Both Physiological and Psychological Stereoscopy from Single Image. International Journal OF Imaging Systems And Technology, 18 (2-3): 146

[12] D. Hoiem, A. Efros, and M. Herbert. Geometric context from a single image. In ICCV(2005).

[13] Julesz Bela, A New Sense for Depth of Field, IEEE Transactions on Pattern Analysis and Machine Intelligence (1987), PAMI-9(4): 523-530. 